4. Johnson, R.J., Nolan, C., Wang, S.P., Shelton, W.R., Blaser, B.S. \& Blaser, M.J. Persistent Campylobacter jejuni infection in a immunocompromised patient. Ann Int Med 1984, 100: 832-834.

5. Ebright, J.R. \& Ryan, L.M. Acute erosive reactive arthritis associated with Campylobacter jejuni induced colitis. Am J Med 1984, 76: 321 - 323.

\section{Hydatid cyst of the neck}

Sir,

Only a few cases of hydatid cyst occurring in the neck area have been reported - the biggest series being 9 cases reported by Touhami et al.' This prompted us to report a further case.

A 25 year old male presented with a swelling on the left side of the lower neck, gradually progressive in size for one year. The swelling was $10 \times 8 \mathrm{~cm}$, situated partially under the left lower part of the sternocledomastoid and was soft in consistency. Oral and ear, nose and throat examination were normal. Fine needle aspiration was carried out which revealed clear fluid and the cytology report was inconclusive. The cyst was excised and the histopathology confirmed it to be echinococcal disease. During follow-up the patient was evaluated for any other site of hydatid, but radiology of chest and ultrasonography of liver, spleen and kidney were normal.

Hydatid cyst develops most commonly in liver $(60 \%)$, lungs $(20 \%)$ and rarely in brain, eye, heart, bone or other internal organs. ${ }^{2}$

The disease presents as a slow growing benign tumour with pressure symptoms according to its site of occurrence. Immunoelectrophoresis is a highly specific test for diagnosis. In the neck area it is very difficult to diagnose hydatid cyst. Even fine needle aspiration cytology is inconclusive unless one submits it for microscopic examination with a high suspicion index. In endemic areas, one should keep in mind the possibility of hydatid cyst when presented with a cystic lump in the neck.

\section{Kundan Kumar Ajay K. Khanna Mahendra K. Misra Department of Surgery, Institute of Medical Sciences, Banaras Hindu University, Varanasi 221005 , India.}

\section{References}

1. Touhami, M., Benkirane, M. \& Ouazzani, H. Cervicofacial hydatid cyst. A series of nine cases. Rev Laryngol Otol Rhinol (Bord) 1985, 106: 187-190.

2. Braud, A. Infectious Disease and Medical Microbiology, 2nd ed. W.B. Saunders, Philadelphia, 1986, pp. 945.

\section{Chilaiditi's syndrome presenting as unexplained tender hepatomegaly}

Sir,

Hepatodiaphragmatic interposition of intestine (HDI; Chilaiditi's syndrome) is a condition when part of the intestine is interposed between the liver and the right dome of the diaphragm. It is a rare condition, the incidence varying between $0.006 \%$ to $0.2 \%$, depending on the age and sex of the patients.' Recently, we have come across an unusual case of HDI mimicking amoebic liver abscess.

A 46 year old labourer was admitted with moderate to severe, throbbing, non-radiating pain in the right upper quadrant of the abdomen of 8 days duration, low grade, intermittent fever and anorexia. Physical examination revealed a firm, smooth and tender liver palpable $15 \mathrm{~cm}$ below the subcostal arch in the right midclavicular plane. The percussion note over the liver was tympanitic in character which merged with the note over the chest. The patient was diagnosed as having an amoebic liver abscess, and was treated with metronidazole.

Complete haemogram and liver function tests were normal. The routine stool examination for Entamoeba histolytica was negative. The plain X-ray of the abdomen taken in the erect position revealed gas under the right diaphragm with a horizontal fluid level. Ultrasonography and contrast enhanced computed tomography confirmed the colonic interposition between the liver and the right dome of the diaphragm. The liver was normal and was pushed downward and medially by the colon. During his hospital stay, he remained afebrile, although the tender hepatomegaly persisted.

HDI is generally described as an asymptomatic and clinically silent syndrome. However, it is of interest to radiologists since it has to be differentiated from the various causes of 'air under the diaphragm'.2 This condition may occur transiently (sliding type), however adhesions may cause persistence of the interposition. ${ }^{3}$ The interposed organ is hepatic flexure of the colon in mos cases but small intestine and omentum may also be present. Multiple factors, discussed at length in an earlier report, ${ }^{4}$ are implicated in the causation of interposition.

This condition may not be entirely clinically irrelevant, as symptoms such as diffuse abdominal pain, nausea, vomiting, flatulence, constipation, shortness of breath and pain in the substernal area may occur. ${ }^{3}$ Various authors have emphasized that abdominal tenderness and displacement of the liver to the left side of the abdomen may be observed. However, a moderately large palpable liver of $15.0 \mathrm{~cm}$ with tenderness over the right hypochondrium, as observed in the present report, has not been previously described. Since Chilaiditi's syndrome is relatively frequent in the rural population and those consuming a vegetarian diet, for example, the Mediterranean region $^{5}$ and Siberia, ${ }^{6}$ it should theoretically be more prevalent in the Indian subcontinent, though no such epidemiological study has been reported from this area. Hence, it is rational to suspect HDI, when unexplained hepatomegaly (with or without tenderness) is encountered.

D.K. Mukhopadhyay S. Srikant

A. Misra

B. Bhatla

R. Uppal

Department of Medicine and 'Radio-Diagnosis, All India Institute of Medical Sciences, New Delhi-110029,

India. 